\title{
The Practice of Cerebral CT at Abidjan: Advocacy for the Implementation of Guidelines
}

\author{
N'goran Kouamé*, Sorel Fotso Manewa, Anne Marie N'goan-Domoua, Roger Daniel N'gbesso \\ Service de Radiologie, CHU de Yopougon, Abidjan, Ivory Coast \\ Email: *kngoran@yahoo.fr
}

How to cite this paper: Kouamé, N., Manewa, S.F., N'goan-Domoua, A.M. and N'gbesso, R.D. (2017) The Practice of Cerebral CT at Abidjan: Advocacy for the Implementation of Guidelines. Open Access Library Journal, 4: e3569.

https://doi.org/10.4236/oalib.1103569

Received: March 28, 2017

Accepted: April 30, 2017

Published: May 3, 2017

Copyright $\odot 2017$ by authors and Open Access Library Inc.

This work is licensed under the Creative Commons Attribution International License (CC BY 4.0).

http://creativecommons.org/licenses/by/4.0/

\begin{abstract}
Objectives: The aim of this study was to assess scanning activities at Yopougon University Hospital through its most common application that is the cranio-encephalic CT in order to make proposals for the improvement of its use. Patients and Methods: This was a three-year-cross-sectional study based on cranio-encephalic CT scans performed at Yopougon University Hospital from January 2011 to December 2013. All CT scans were performed on a TOSHIBA 64-bar scanner with or without injection of iodinated contrast agent. The variables studied were the epidemiological-clinical elements (age, gender and indications), the exploration technique, the overall results and the various pathologies discovered. Results: The mean age of our patients was 35.4 years with a predominance of children (age group of 0 and 14 years). The sex ratio was 1.38. Motor deficits (19.3\%) and head trauma (17.5\%) was the main indication of cranio-encephalic scanners. We performed 221 examinations without injection of iodinated contrast agent (36.8\%) and 379 examinations with injection of iodinated contrast agent (63.2\%). We recorded 298 normal findings and 302 pathological findings that are $49.7 \%$ and $50.3 \%$ respectively. Strokes were the predominant pathology found on the cranio-encephalic scanners (43.7\%) followed by traumatic pathology (20.9\%). Conclusion: The rate of normal findings for cranio-encephalic scanners was very high (49.7\%). Pathological findings were dominated by strokes (43.7\%) and traumatic pathology (20.9\%). Infectious pathology was paradoxically rare (3\%). A more rigorous prescription of cranio-encephalic scanners based on a well-conducted clinical examination and the guides of good use of imaging examinations (guidelines) could help to reduce the rate of normal scanner at Yopougon University Hospital.
\end{abstract}

\section{Subject Areas}

Pathology 


\section{Keywords}

Cranio-Encephalic CT Scan, Activity Assessment, Scanning Practice, Africa

\section{Introduction}

La tomodensitométrie (TDM) ou scanographie, encore appelée computerized tomography (CT) par les Anglo-saxons est une technique d'imagerie radiologique permettant d'obtenir des coupes axiales reconstruites à partir du coefficient d'atténuation des rayons $\mathrm{X}$ dans le volume exploré. La TDM sert à mesurer l'absorption des rayons $\mathrm{X}$ au sein d'un volume exploré, à l'aide de multiples incidences d'un faisceau de rayons $\mathrm{X}$ [1] [2].

Son utilisation médicale dans les années 1970 a révolutionné le diagnostic médical en particulier dans le domaine de la neurologie. Grâce à l'amélioration continue de ses performances techniques, la TDM a connu un essor considérable, depuis ces 20 dernières années, justifié par son intérêt diagnostique [2].

Aujourd'hui, partout dans le monde entier, le scanner et l'imagerie par résonance magnétique constituent les examens d'imagerie incontournables dans l'exploration cranio-encéphalique. L'Afrique subsaharienne en général et la Côte d'Ivoire en particulier n'est pas en reste de cette évolution.

Si l'IRM haut champ n'est pas encore disponible dans les hôpitaux publics en Côte d'Ivoire, ce n'est pas le cas de la tomodensitométrie qu'on peut retrouver dans tous les Centres Hospitalo-Universitaires ( $\mathrm{CHU})$ de la Côte d'ivoire. A Abidjan, le $\mathrm{CHU}$ de Yopougon constitue la référence puisqu'elle possède le scanner le plus performant (64 barrettes). Comment ce scanner est-il utilisé? quel est son apport réel dans le diagnostic des pathologies neurologiques? Notre étude a pour but de faire le bilan des activités du scanner du CHU de Yopougon à travers son application la plus fréquente qu'est la TDM cranio-encéphalique afin de faire des propositions pour l'amélioration de son utilisation.

\section{Matériel et Méthode}

Notre étude rétrospective transversale s'est déroulée sur une période de trois (03) ans allant de Janvier 2011 à Décembre 2013 au service de radiologie du CHU de Yopougon. Elle a concerné tous les patients chez qui au moins un scanner cranio-encéphalique a été réalisé. Etaient inclus dans notre étude tous les patients qui avaient un compte rendu scanographique cranio-encéphalique complet mentionnant l'âge, le motif de demande (indication), le protocole technique de réalisation de l'examen, les résultats détaillés et la pathologie mise en évidence. Tous les scanners ont été réalisés sur un appareil scanographique de marque TOSHIBA de 64 barrettes avec ou sans injection de produit de contraste iodé. Les variables étudiées étaient les éléments épidémio-cliniques (âge, sexe et indications), la technique d'exploration, les résultats globaux et les différentes pathologies découvertes. Les données ont été décrites en utilisant les fréquences et les pourcentages. La saisie des données a été faite sur le logiciel WORD 2007. 
Les tableaux et les graphiques ont été conçu sur le logiciel EXCEL 2007.

\section{Résultats}

Les scanners cranio-encéphaliques représentaient l'activité principale du service du scanner du CHU de Yopougon (67\%) suivi du scanner du rachis (20\%). L'âge moyen de nos patients était de 35.4 ans avec une prédominance des enfants (classe d'âge entre 0 et 14 ans). Chez les adultes la classe d'âge entre 45 et 59 ans était la plus concernée par la réalisation des scanners cranio-encéphaliques.

Sur un total de 600 patients, 348 étaient de sexe masculin et 252 de sexe féminin, d'où un sex ratio de 1.38. Soit environ 4 hommes pour 3 femmes ont bénéficié d'un scanner cranio-encéphalique pendant ces 3 années. Les déficits moteurs (19.3\%) et les traumatismes crâniens (17.5\%) constituaient les principales indications des scanners cranio-encéphaliques (Table 1). Les autres indications étaient constituées par le syndrome infectieux, les troubles de la conscience, les troubles de la vigilance, les agitations et les troubles du comportement. Nous avons réalisé 221 examens sans injection de produit de contraste iodé (36.8\%) et 379 examens avec injection de produit de contraste iodé (63.2\%). Nous avons dénombré 298 résultats normaux et 302 résultats pathologiques soit respectivement $49.7 \%$ et $50.3 \%$. Les accidents vasculaires cérébraux (Table 2) constituaient la pathologie prédominante retrouvée à la TDM CE (43.7\%) suivis des traumatismes (20.9\%). Les malformations cérébrales étaient dominées par les malformations vasculaires, les anévrysmes cérébraux à hauteur de 57.1\%. Les accidents vasculaires cérébraux (Figure 1) étaient dominés par les accidents vasculaires ischémiques (62.7\%). Nous n'avons pas retrouvé de thrombophlébite dans notre série. Les fractures et les contusions hémorragiques représentaient l'essentiel des lésions élémentaires de la pathologie traumatique (Figure 2) avec respectivement une proportion de $42.9 \%$ et $22.1 \%$. Les tumeurs sus tentorielles étaient prédominantes dans notre étude (47.4\%) suivies des

Table 1. Répartition des patients en fonction de l'indication du scanner.

\begin{tabular}{ccc}
\hline & EFFECTIFS & POURCENTAGE \\
\hline Déficit moteur & 116 & 19.3 \\
Traumatismes crâniens & 105 & 17.5 \\
Suspicion d'AVC & 96 & 16 \\
Céphalées diverses & 89 & 14.8 \\
Examens sans indications & 51 & 8.5 \\
Convulsions & 47 & 7.8 \\
Malformations cérébrales & 45 & 7.5 \\
Contrôle après drainage & 18 & 3 \\
Recherche et bilan de masse tumorale & 11 & 1.8 \\
Autres indications* & 22 & 3.7 \\
TOTAL & 600 & 100 \\
\hline
\end{tabular}

*les autres indications étaient constituées par les syndromes infectieux, les troubles de la conscience, les troubles de la vigilance, les agitations et les troubles du comportement. 
Table 2. Répartition des patients selon la pathologie mise en évidence.

\begin{tabular}{ccc}
\hline & EFFECTIF & POURCENTAGE \\
\hline Pathologie malformative & 7 & 1.9 \\
Accidents vasculaires cérébraux & 161 & 43.7 \\
Pathologie traumatique & 77 & 20.9 \\
Pathologie tumorale & 19 & 5.2 \\
Pathologie infectieuse & 12 & 3.3 \\
Pathologie séquellaire & 52 & 14.1 \\
Hydrocéphalie & 40 & 10.9 \\
TOTAL & $368^{*}$ & 100 \\
\hline
\end{tabular}

* plusieurs pathologies ont été trouvées chez le même patient.

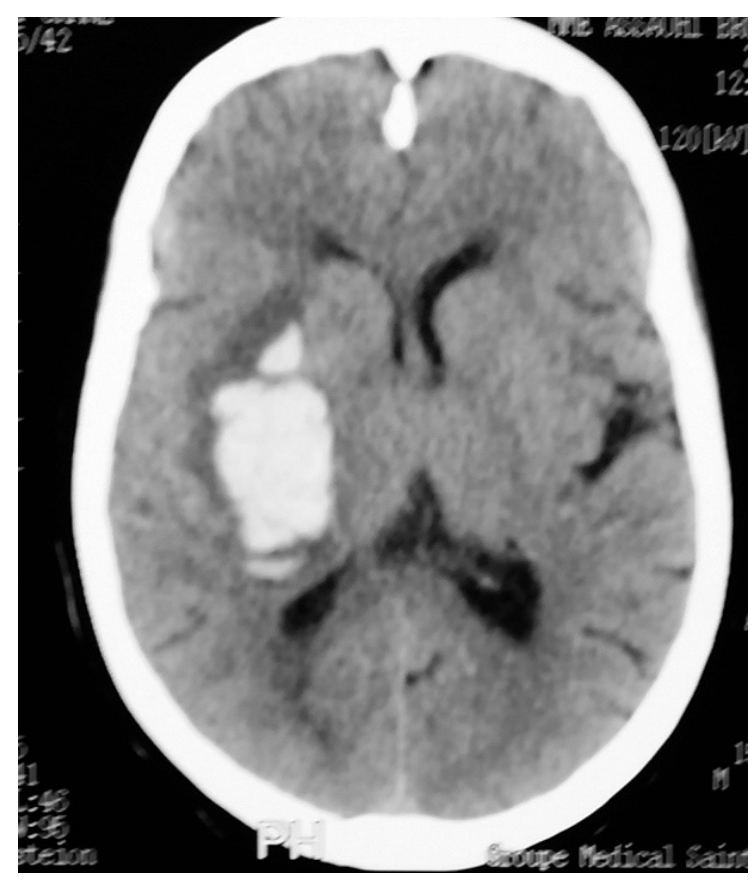

Figure 1. Patient de 60 ans hypertendu connu. Hématome intra-parenchymateux droit (Flèche) entrant dans le cadre d'un AVC hémorragique.

tumeurs (Figure 3) de la ligne médiane et de celles sous tentorielles à hauteur de $26.3 \%$ chacune. L'encéphalite pré suppurative représentait la pathologie infectieuse (Figure 4) dominante (41.7\%). La pathologie séquellaire était dominée par les atrophies cortico-sous-corticales (79.1\%).

\section{Discussion}

La population de notre étude était jeune. L'âge moyen de nos patients était de 35.4 ans. Diallo [3], au Burkina-Faso a objectivé une moyenne d'âge de 33 ans. Cette jeunesse des patients de notre étude pourrait s'expliquer par le fait que la classe d'âge entre 0 et 14 ans était prédominante (22.3\%). En effet, plusieurs auteurs Africains ont utilisé le scanner pour explorer les pathologies cranio-encéphaliques de l'enfant. ZEH au Cameroun [4], en 2006 a exploré en 6 ans, 70 enfants uniquement pour la recherche des complications des méningites. 


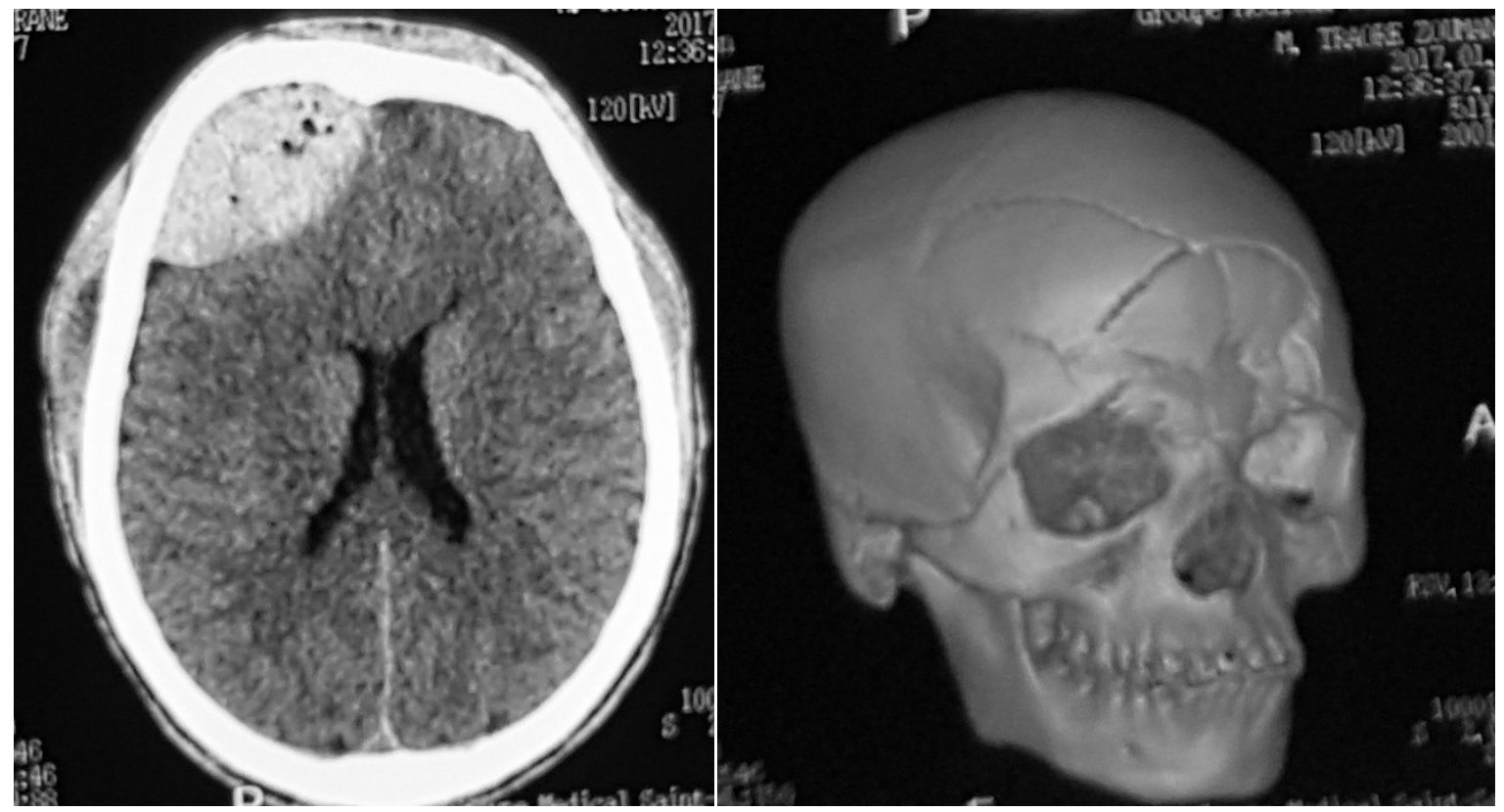

Figure 2. Patient de 22 ans. Traumatisme crânien par chute d'une moto avec réception sur la face et perte de connaissance initiale. Scanner en coupe axiale en fenêtre parenchymateuse et reconstruction 3D montrant un hématome extra-dural frontal droit (Flèche) avec fractures complexes cranio-faciales.

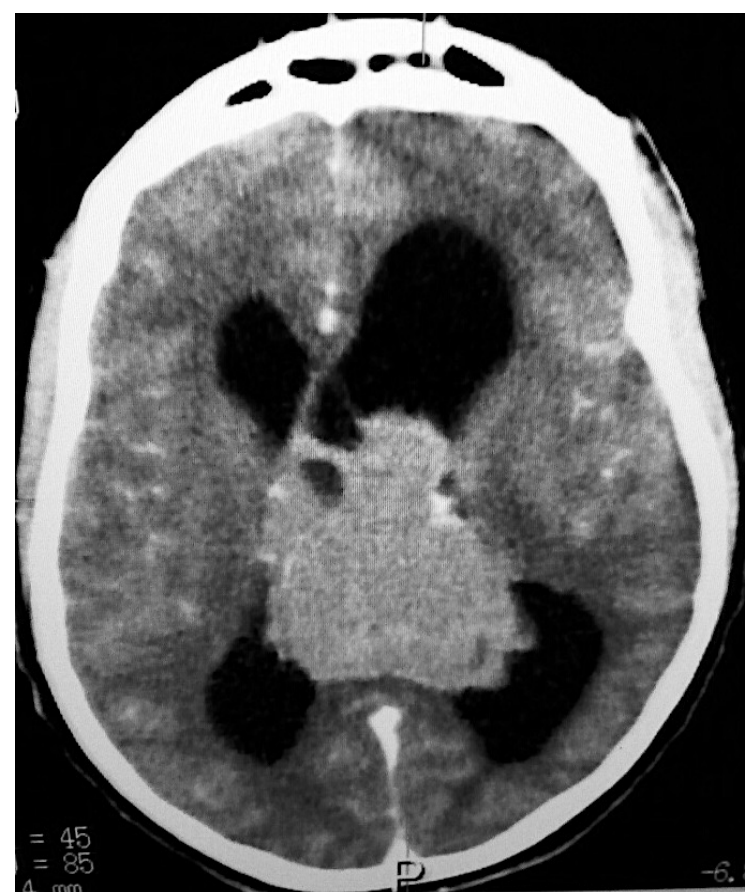

Figure 3. Patiente de 20 ans. Troubles cognitifs. Scanner avec injection en coupe axiale. Tumeur du $3^{\text {ème }}$ ventricule (étoile) responsable d'une hydrocéphalie biventriculaire (Flèche).

N'goan-Domoua et al. [5] à Abidjan ont utilisé également le scanner pour explorer 24 cas de suppurations intra-crâniennes chez l'enfant en 2 ans. Cette tendance Africaine à explorer les pathologies cranio-encéphaliques de l'enfant au scanner n'est pas la même que dans les pays développés. En effet, l'enfant ayant 


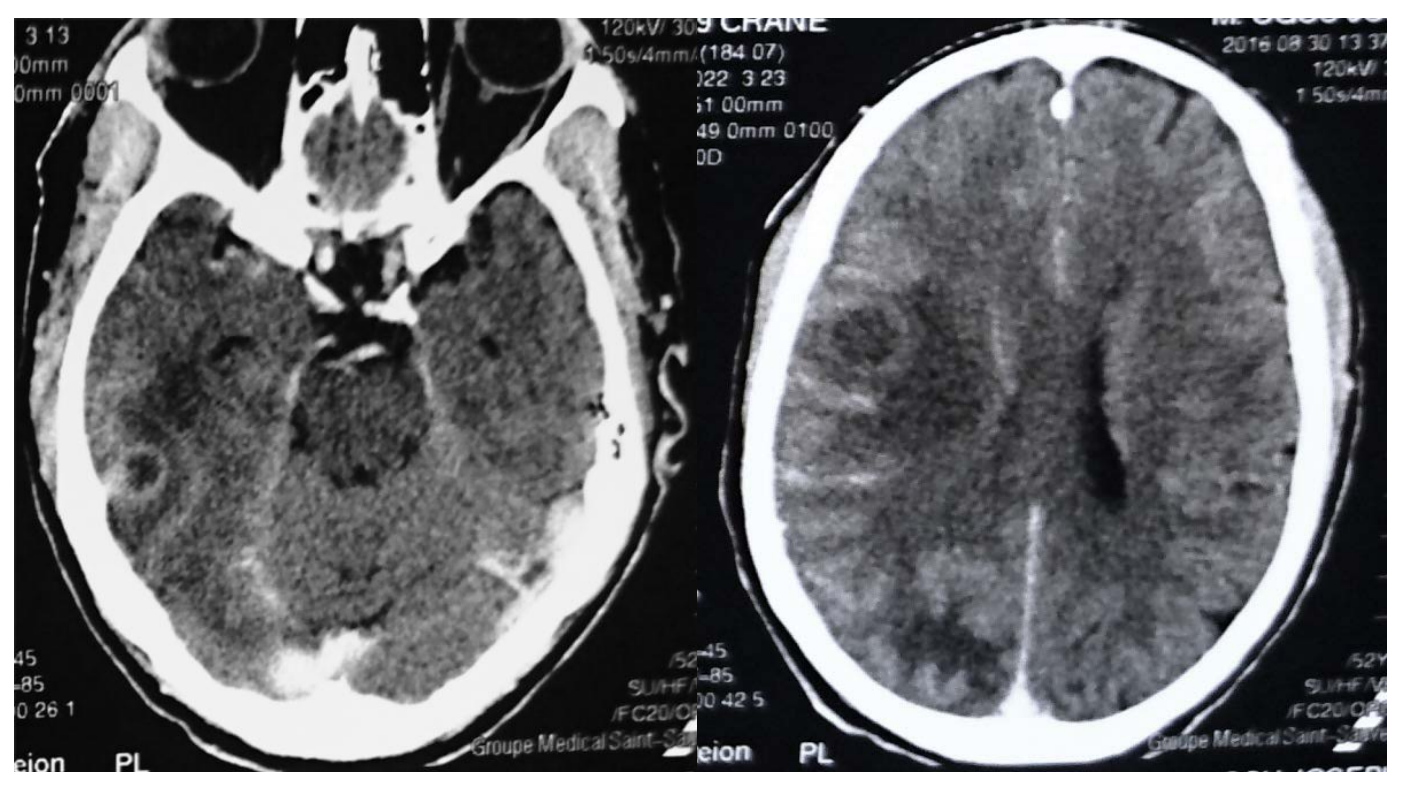

Figure 4. Patient de 52 ans. Déficit moteur d'installation progressive. Scanner avec injection de PCI: prise de contraste annulaire temporale droite et pariétale droite cernée par une plage hypodense d'œdème: abcès cérébraux.

un organisme en croissance, il est sensible aux effets nocifs des rayons $\mathrm{X}$ avec pour corollaire le développement des cancers. L'IRM et l'échographie transfontanellaire sont donc préférés au scanner dans les pays développés [6]. Cette pratique Africaine pourrait s'expliquer par l'insuffisance du plateau technique. Car bien que les appareils d'échographie soient disponibles, leur utilisation est limitée par la fermeture de la fontanelle bregmatique à partir de 18 mois. Par ailleurs les hôpitaux Africains ne sont pas équipés d'appareil d'IRM haut champ.

Sur un total de 600 patients, 348 étaient de sexe Masculin et 252 de sexe féminin, d'où un sex ratio 1.38. Nos résultats sont sensiblement les mêmes que ceux de Diallo au Burkina [3] qui trouve un sex ratio de 2.08. Cette prédominance du sexe masculin pourrait s'expliquer par la forte proportion de traumatisme cranio-encéphalique (TCE) dans notre étude. En effet, les hommes sont plus exposés aux TCE à cause du fait qu'ils sont plus actifs et prennent plus de risques au dehors tandis que la principale activité féminine est domestique [5].

Dans notre étude, Les déficits moteurs (19.3\%) et les traumatismes crâniens (17.5\%) constituaient les principales indications des scanners cranioencéphaliques. Ces indications sont en rapport, en termes de fréquence avec les pathologies cranio-encéphaliques couramment rencontrées dans la pratique quotidienne. Les accidents vasculaires cérébraux, responsables de la plupart des déficits moteurs et les traumatismes crâniens étant les pathologies les plus fréquentes tant en Afrique que dans les pays développés. Selon Manessiez [7], les traumatismes crâniens représentent un véritable problème de santé publique du fait de leur fréquence. Ils sont responsables de près de $7 \%$ des passages aux urgences dans une étude épidémiologique nord-américaine et constituent en 
France la première cause d'hospitalisation dans le secteur public parmi les 20 diagnostics à caractère d'urgence. Les traumatismes crânio-encéphaliques étaient les indications majoritaires (17.31\%) selon Tchaou au TOGO en 2016 [8].

Dans notre étude, $63.2 \%$ des patients ont bénéficié d'un scanner avec injection de produit de contraste iodé. Ce qui veut dire qu'ils ont eu 2 acquisitions. Selon Jankowski [2], l'injection de produit de contraste est utile dans l'étude des structures vasculaires, pour différencier pathologie inflammatoire et tumorale et pour la recherche de localisations secondaires. Au regard des indications qui sous-tendent la réalisation du scanner, nous pensons que cette proportion d'injection de produit de contraste est élevée. En effet, les indications sont dominées par les suspicions d'AVC et les traumatismes crâniens. De plus, la proportion élevée d'enfant dans notre étude devrait faire éviter une deuxième acquisition quand nous savons que les scanners multi-barrettes sont très irradiants. Nous sommes en accord avec Tchaou [8] qui stipule que les indications et la réalisation des scanners chez l'enfant au TOGO n'est pas totalement conforme avec les guides de bon usage des examens d'imagerie. Il estime également que les doses délivrées aux enfants excèdent les normes internationales.

Dans notre étude, les résultats du scanner étaient normaux dans $49.7 \%$ des cas. Le taux de résultats normaux apparait très élevés en comparaison avec les données de la littérature. En effet Kouamé et al. [9] à Abidjan ont obtenu 31\% de TDM normale dans leur étude sur la tomodensitométrie dans la recherche étiologique des comas. Mais nos résultats étaient inférieurs à ceux de ARMOR en Tunisie [10] qui a trouvé $77 \%$ de scanner normal dans son étude sur le scanner des traumatismes crâniens. Ce constat amène l'auteur à se demander s'il fallait pratiquer le scanner au cours des traumatismes cranio-encéphaliques bénins. Ce constat nous ramène au débat de la suprématie de la clinique sur les examens paracliniques. Quand on sait que le scanner coute très cher dans un pays pauvre comme le nôtre, il serait plus judicieux de se remettre à la clinique en particulier à la classification de Master [11] qui détermine quand le scanner est utile en cas de traumatisme cranio-encéphalique. Cette classification définit 3 groupes à risque croissant de complications intracrâniennes: groupe 1 risque faible, groupe 2 risque modéré, groupe 3 risque élevé. Le groupe 1 ne devrait jamais bénéficier de scanner.

Il est également nécessaire de souligner la suprématie de l'IRM dans l'exploration des pathologies cérébrales. Certains auteurs ont démontré que les pathologies axonales lors des traumatismes cranio-encéphaliques ne sont visibles qu'à l'IRM. Cette dernière est également plus sensible dans les accidents vasculaires cérébraux au stade précoce et dans d'autres pathologies telles que l'épilepsie temporale, les pathologies infectieuses et inflammatoires au stade de début et le tumeurs hypophysaires [12] [13].

La moitié des résultats des scanners cranio-encéphaliques réalisés dans notre étude étaient pathologiques. Les pathologies que nous avons mises en évidence étaient dominées par les accidents vasculaires cérébraux (43.7\%) suivis de très 
loin par la pathologie traumatique et les hydrocéphalies (10.9\%). Les pathologies tumorale et infectieuse venaient en dernière position à des taux respectifs de $5.2 \%$ et $3.3 \%$.

Les accidents vasculaires cérébraux quoique prédominants dans l'étude de Nikiéma à Bobo-Dioulasso [14] avaient une fréquence plus faible comparée à la nôtre (18.1\%). Cette faiblesse pourrait s'expliquer par le fait que son étude, basée sur l'évaluation de toute l'activité scanographique sur une période de 3 ans alors que notre étude s'est uniquement focalisée sur le scanner cranio-encéphalique.

Cette prédominance des AVC pourrait s'expliquer par le fait que, selon Nkouna [15], les accidents vasculaires cérébraux tendent à devenir un véritable problème de santé publique, même dans les pays en développement en raison de leur fréquence et de leur mortalité élevée.

Ces AVC étaient dominés par les accidents vasculaires ischémiques (62.7\%) suivis des accidents hémorragiques (37.3\%). Nos résultats concordent avec ceux de N'goran [16], à l'institut de cardiologie d'Abidjan. Selon ses résultats, l'AVC ischémique était le type lésionnel dominant constituant plus de $3 / 4$ des lésions (84.1\%) dont 65\% de lésion ischémique au scanner cérébral et $19.1 \%$ de scanner normal et les AVC hémorragiques $15.9 \%$ des cas. Cette suprématie de l'AVC ischémique sur l'AVC hémorragique est également mise en évidence dans les études de Diagana [17] en Mauritanie et de Ikeh [18] au Nigéria. La thrombophlébite décrite par certains auteurs n’a pas été observée dans notre étude. Elle apparaît cependant rare au regard des résultats mis en évidence dans la littérature. Elle représente $1.4 \%$ dans l'étude de Zeh [4] et 1.6\% dans celle de Etoundi [19].

La pathologie traumatique cranio-encéphalique vient en $2^{\text {ème }}$ position en termes de fréquence (20.9\%), après les AVC, dans notre étude. Dans notre étude, les fractures étaient moindres en terme de proportion (42.9\%) par rapport aux saignements $(57.1 \%)$. Ces saignements étaient représentés par ordre de fréquence par les contusions hémorragiques (22.1\%), les hématomes sous duraux (16.8\%), les hématomes intra-parenchymateux (10.4\%) et les hématomes extra-duraux $(7.8 \%)$. Les associations contusion hémorragique et hématome intra parenchymateux ont dominé dans les lésions cérébrales avec $40 \%$ chacune. L'association hémorragie méningée et hématome extra dural ont prédominé dans les lésions péri-cérébrales. Dans l'étude de Sidibé [20], une lésion cérébrale ou péri-cérébrale était retrouvée dans $88.2 \%$ alors que dans $24.8 \%$, il s'agissait d'une lésion osseuse ou du scalp. La lésion était cérébrale dans $58.8 \%$, péri-cérébrale dans $32.6 \%$, mixte dans $8.6 \%$. Les hématomes extra-duraux trouvés dans notre étude étaient dans une proportion plus faible que celle mise en évidence par N'GBESSO [21] à Abidjan en 2004 sur étude ayant regroupée 297 dossiers scanographiques de traumatisme cranio-encéphalique: $12.6 \%$ dans l'étude de N'GBESSO contre 7.8\% dans notre étude.

Les hydrocéphalies avec une proportion de $10.9 \%$ venaient en $3^{\text {ème }}$ position après les accidents vasculaires cérébraux et les traumatismes en terme de fréquence. Cette élévation du taux d'hydrocéphalie pourrait s'expliquer par le 
nombre important des enfants dans notre effectif. En effet selon la littérature, les hydrocéphalies de l'enfant peuvent être d'origine malformative [22], tumorale [23] ou en rapport avec une sténose de l'acqueduc de Sylvius [24].

Les hydrocéphalies de l'enfant en Afrique subsaharienne sont le plus souvent liées aux séquelles de méningites responsables de fibrose de l'aqueduc de Sylvius [4] [25].

A Abidjan, en plus de l'étiologie post-méningitique, Broalet [23] pointe du doigt les tumeurs. Elle représentait 5.3\% des pathologies retrouvées au scanner cranio-encéphalique. Ces tumeurs intéressaient à la fois les adultes et les enfants. Elles étaient dominées par les tumeurs sus tentorielles et extra-axiales en particulier les méningiomes (21\%). Nos résultats sont superposables à ceux trouvés par Doumbia à Abidjan [26]. Dans son étude concernant les explorations TDM des épilepsies, les causes tumorales représentaient $6.7 \%$.

La pathologie infectieuse était paradoxalement la pathologie la moins fréquemment rencontrée dans notre étude quand on sait que notre étude a eu lieu dans un pays tropical et à l'ère de la pandémie du VIH-SIDA. Elle représentait environ $3 \%$ des pathologies explorées dans notre étude et était dominée par l'encéphalite pré suppurative (41\%), l'abcès intracérébral (25\%) et péri-cérébral (25\%). Nos résultats concordent avec ceux de Mapoure [27] à Dakar qui met en évidence $8.8 \%$ de causes infectieuses dans son étude qui évaluait les étiologies des comas au scanner.

\section{Conclusion}

La TDM CE représente la principale activité scanographique au CHU de Yopougon $(67 \%)$. Les hommes $($ sex ratio $=1.4)$ jeunes (âge moyen $=35.5 \%)$, présentant des déficits moteurs (19.3\%) ou victimes de traumatisme crâniens (17.5\%) étaient les plus concernés. La plupart des TDM CE a été réalisée avec injection de produit de contraste (63\%). Le taux de résultats normaux était très élevé (49.7\%). Les résultats pathologiques étaient dominés par les AVC (43.7\%) et la pathologie traumatique (20.9\%). La pathologie infectieuse était paradoxalement rare (3\%). Une prescription plus rigoureuse des scanners cranioencéphaliques basée sur un examen clinique bien menée et les guide de bon usage des examens d'imagerie pourrait contribuer à réduire le taux de scanner normal au CHU de Yopougon.

\section{References}

[1] Hubert, J., Descotes, J.L. and Blum, A. (2003) Tomodensitométrie. Progrès en Urologie, 13, 783-794.

[2] Jankowski, A. and Ferretti, G. (2010) Tomodensitométrie volumique: Principe, paramètres. Revue des Maladies Respiratoires, 27, 964-969.

https://doi.org/10.1016/j.rmr.2010.09.004

[3] Diallo, O., Kabre, A., Lougué-Sorgho, L.C., Napon, C., Ouéda, M., Bamouni, Y.A., Zoungrana, R., Napon, A.M., Ouattara, B. and Cissé, R. (2009) Imagerie de suppurations collectées cranio-encéphaliques au chu Yalgado Ouedraogo: A propos de 40 cas. Journal de Radiologie, 90, 1466-1467. 
https://doi.org/10.1016/S0221-0363(09)75752-X

[4] Zeh, O.F., Guegang, G.E., Moifo, B., Nguefack, S., Nchagnouot, M.F., Nwatsock, J.F., Mbassi, A.H., Monabang, Z.C. and Gonsu, F.J. (2014) Aspects tomodensitométriques des complications cérébrales des méningites bactériennes de l'enfant à Yaoundé. Journal Africain d' Magerie, 6, 3-13.

[5] N'goan-Domoua, A.M., Kouamé, N. and N'gbesso, R.D. (2009) Apport de la tomodensitométrie dans l'exploration des suppurations intracrâniennes de l'enfant. Revue Internationale des Sciences Médicales, 11, 38-41.

[6] Chateil, J.F., Adamsbaum, C. and Husson, B. (2007) Imagerie en neuropediatrie: Pathologie endocanalaire chez l'enfant. Journal de Radiologie, 88, 1488.

[7] Manessiez, O., Aufort, S., King, J.P., Menjot de Champfleur, N., Benatia, P. and Taourel, P. (2007) Indications du scanner cérébral chez les patients présentant un traumatisme crânien bénin. Journal de Radiologie, 88, 567-571.

[8] Tchaou, M., Gnakadja, G.N., N’timon, B., Sonhaye, L., Amadou, A., AgodaKoussema, L.K., Adjenou, K. and N'dakena, K. (2016) Revue des Doses d'Exposition et des Méthode d'Optimisation en Tomodensitométrie (TDM) de l'Enfant Au Togo. European Scientific Journal, 12, 56-66.

[9] Kouamé, N., Ngoan-Domoua, A.M., Setcheou, A., Konan, A.N., Ngbesso, R.D. and Keita, A.K. (2009) Apport de la tomodensitométrie dans le bilan étiologique des comas non traumatiques à Abidjan. Journal de Radiologie, 90, 1466.

https://doi.org/10.1016/S0221-0363(09)75751-8

[10] Belhaj Amor, M., Ben Salah, M., Babay, H., Ibrahim, H., Tanfous, I. and Balma, A. (2006) Faut-il pratiquer un scanner cérébral chez tout traumatisme crânien bénin? Journal Maghrébin d'Anesthésie-Réanimation et de Médecine d Urgence, 13, 5-8.

[11] Master, S.J., Mc Clean, P.M. and Arcarèse, J.S. (1987) Skull X-Ray Examination after Head Trauma: Recommandations by a Multidisciplinary Panel and Validation Study. The New England Journal of Medicine, 316, 84-91. https://doi.org/10.1056/NEJM198701083160205

[12] Merzoug, V., Kalifa, G. and Gendrel, D. (2001) Place du scanner dans les maladies infectieuses de l'enfant. Médecine Thérapeutique/ Pédiatrie, 4, 291-298.

[13] Yahyaoui, S., Majdoub, S., Zaghouani, H., Yahyaoui, S., Majdoub, S., Zaghouani, H., Ben Fradj, H., Bakir, D., Bouajina, E. and Kraiem, C. (2013) Une localisation exceptionnelle de la tuberculose vertébrale Mal de Pott sous-occipital. The Pan African Medical Journal, 14, 163. https://doi.org/10.11604/pamj.2013.14.163.2450

[14] Nikiema, Z., Sombié, I., Ilboudo, P., Traoré, S.S., Traoré-Zorom, B., Lougué-Sorgho, C. and Cissé, R. (2013) Activités tomodensitométriques au centre hospitalier universitaire SOUROSANOU de Bobo-Dioulasso. Burkina Medical, 17, 95-102.

[15] Kouna Ndouongo, P., Siéméfo, M.A., Kamgang, F.P. and Assengone-Zeh, Y. (2007) Aspects épidémiologiques et évolutifs des accidents vasculaires au centre hospitalier de Libreville (Gabon). AJNS, 26, 12-17.

[16] N'goran, Y.N.K., Traore, F., Tano, M., Kramoh, E.K., Anzoua-Kacou, J.B., Konin, C. and Kacou, M.G. (2015) Aspects épidémiologiques des accidents vasculaires cérébraux (AVC) aux urgences de l'institut de cardiologie d'Abidjan (ICA). The Pan African Medical Journal, 21, 160.

[17] Diagana, M., Traore, H., Bassima, A., Druet-Cabanac, M., Preux, P.M. and Dumas, M. (2002) Apport de la tomodensitométrie dans le diagnostic des accidents vasculaires cérébraux à Nouakchott, Mauritanie. Médecine Tropicale, 62, 145-149.

[18] Ikeh, V.O., Nwosu, M.C. and Nwabueze, A.C. (1988) The Epidemiology of Stroke in Nigerians: A Study of 328 Cases. Cardiologie Tropicale, 14, 63-67. 
[19] Owono Etoundi, P., Esiéne, A., Bengono Bengono, R., Amengle, L., Afane Ela, A. and Ze Minkande, J. (2015) Aspects épidémiologiques de la maladie thromboembolique veineuse à Yaoundé. Health Sciences and Diseases, 16, 1-4.

[20] Sidibé, S., Diallo, A., Touré, M., Tchamko Djeutcheu, F.R. and Traoré, I. (2005) Apport de la tomodensitométrie dans la prise en charge des traumatismes cranioencéphaliques à Bamako. Mali Médical, 10, 33-36.

[21] N'Gbesso, R.D., N'Goan-Domoua, A.M., Ould Beddi, M., Yoman, A.M.F. and Kéita, A.K. (2004) Traumatismes crâniens en Côte d'Ivoire: Evaluation TDM de 297 cas. Médecine d' Afrique Noire, 51, 595-601.

[22] Berrouyne, A., Benmoussa, R., Chbani, K., Salam, S. and Ouzidane, L. (2016) Les hydrocéphalies malformatives de l'enfant: étude rétrospective à propos de 50 cas. Journal of Neuroradiology, 43, 94-95. https://doi.org/10.1016/j.neurad.2016.01.063

[23] Broalet, M.Y.E., Haidara, A., Zunon-Kipre, Y., N'dri, O.D., N'da, H., Jibia, A., Kakou, M., Varlet, G. and Bazeze, V. (2007) Approche diagnostique des tumeurs cérébrales chez l'enfant-Experience du service de neurochirurgie du CHU de Yopougon Abidjan. AJNS, 26, 27-38.

[24] Aldea, S. and Gaillard, S. (2009) Diagnostic et traitement des sténoses de l'aqueduc décompensées. Neurologie.com, 1, 53-55.

[25] Ba, M.C., Kpelao, E., Thioub, S.M., Kouara, M., Thiam, A.B., Ndoye, N., Sakho, Y. and Badiane, S.B. (2012) Hydrocéphalie post-méningitique du nourrisson à Dakar. AJNS, 31, 1-5.

[26] Doumbia, M., Kouassi, L., Aka Diarra, E., Kouame-Assouan, A.E., Tanoh, C., Yapo, F.B. and Douayoua-Sonan, T. (2008) Apport de la tomodensitométrie cérébrale dans la prise en charge des épilepsies de l'adulte en Côte d'Ivoire. Epilepsies, 20, 290-293.

[27] Mapoure, N.Y., Sène Diouf, F., Ndiaye, M., Mbatchou Ngahane, H.B., Doumbe, J., Toure, K., Mboup, B., Thiam, A., Diop, A.G. and Ndiaye, M.M. (2009) Etude longitudinale prospective des comas en milieu neurologique africain: Expérience de Dakar, Sénégal. Revue Médicale de Bruxelles, 30, 163-169.

Submit or recommend next manuscript to OALib Journal and we will provide best service for you:

- Publication frequency: Monthly

- 9 subject areas of science, technology and medicine

- Fair and rigorous peer-review system

- Fast publication process

- Article promotion in various social networking sites (LinkedIn, Facebook, Twitter, etc.)

- Maximum dissemination of your research work

Submit Your Paper Online: Click Here to Submit

Or Contact service@oalib.com 